\title{
Evidence for Functional Diversification Within a Fungal NEP1-Like Protein Family
}

\author{
Parthasarathy Santhanam, ${ }^{1}$ H. Peter van Esse, ${ }^{1}$ Isabell Albert, ${ }^{2}$ Luigi Faino, ${ }^{1}$ Thorsten Nürnberger, ${ }^{2}$ and \\ Bart P. H. J. Thomma ${ }^{1,3}$ \\ ${ }^{1}$ Laboratory of Phytopathology, Wageningen University, Droevendaalsesteeg 1, 6708 PB Wageningen, The Netherlands; \\ ${ }^{2}$ Center for Plant Molecular Biology-Plant Biochemistry, University of Tübingen, D-72076 Tübingen, Germany; ${ }^{3}$ Centre for \\ BioSystems Genomics, P.O. Box 98, 6700 AB Wageningen, The Netherlands
}

Submitted 13 September 2012. Accepted 5 October 2012.

In this study, we functionally analyzed the gene family encoding necrosis- and ethylene-inducing-like proteins (NLP) of the vascular wilt pathogen Verticillium dahliae. We show that the composition of the $N L P$ gene family varies little among $V$. dahliae isolates. The cytotoxic activity of NLP family members of a tomato-pathogenic $V$. dahliae strain was determined, demonstrating that only two of the seven NLP induced plant cell death. The genes encoding these cytotoxic NLP were found to be induced in $V$. dahliae upon colonization of tomato. Interestingly, targeted deletion of either of the two genes in $V$. dahliae significantly compromised virulence on tomato as well as on Arabidopsis plants, whereas deletion of only one of the two genes affected virulence on Nicotiana benthamiana. This could be attributed to differential induction of the two NLP genes in $V$. dahliae upon $N$. benthamiana colonization, revealing that the in planta induction of $N L P$ genes varies between plant hosts. Intriguingly, one of the $N L P$ genes appears to also affect vegetative growth and conidiospore production, because the corresponding deletion strain produced significantly fewer conidiospores and developed extensive aerial mycelium. In conclusion, we demonstrate that the expanded $V$. dahliae NLP family shows functional diversification, revealing not only differential cytotoxicity between family members but also that the cytotoxic NLP play a role in vegetative growth and asexual reproduction in addition to their contribution to virulence.

Microbial pathogens employ secreted effectors to modulate the physiology of their intended host, often through deregulation of host defenses, in order to establish disease (de Jonge et al. 2011). Whereas particular effectors remain extracellular, others are able to enter the host cell cytoplasm (Dodds and Rathjen 2010). Typically, effectors are species-specific molecules that are often small, cysteine-rich peptides with no homology to previously characterized proteins. However, some effectors seem to be conserved across species (Thomma et al. 2011). An example of such conserved effectors is provided by

Corresponding author: B. P. H. J. Thomma; Telephone: 0031-317-484536; Fax: 0031-317-483412; E-mail: bart.thomma@wur.nl

* The $\boldsymbol{e}$-Xtra logo stands for "electronic extra" and indicates seven supplementary figures and one supplementary table are published online. Also, Figures 1, 3, 4, 5, and 7 appear in color online.

(C) 2013 The American Phytopathological Society the fungal LysM effectors, members of which enhance pathogen virulence by suppression of chitin-triggered immunity in a number of plant pathogens (Bolton and Thomma 2008; de Jonge et al. 2010; Kombrink et al. 2011; Marshall et al. 2011; Mentlak et al. 2012). Another group of conserved effectors are the necrosis- and ethylene-inducing-like proteins (NLP) (Gijzen and Nürnberger 2006).

The first member of the NLP family has been identified as NEP1, found in culture filtrates of the fungus Fusarium oxysporum (Bailey 1995). However, it is presently recognized that NLP are widely distributed, because they have been identified in many pathogenic bacteria, fungi, and oomycetes, and especially in this latter group of organisms, the NLP family is significantly expanded (Dong et al. 2012; Gijzen and Nürnberger 2006; Pemberton and Salmond 2004). NLP typically share a conserved NPP1 domain, containing a heptapeptide "GHRH DWE" motif (Fellbrich et al. 2002). NLP generally induce immune responses and cell death in dicotyledonous plants and, based on crystal structure analysis and mutagenesis, it has been proposed that NLP can function as cytolytic toxins that induce plasma membrane leakage, thus causing cytotoxicity (Ottmann et al. 2009; Qutob et al. 2006). Several reports have shown that NLP contribute to pathogen virulence (Mattinen et al. 2004; Pemberton et al. 2005), although targeted deletion of $N L P$ genes in other species did not appear to affect pathogen virulence (Motteram et al. 2009; Staats et al. 2007). Thus, likely, NLP play different roles in different pathogens and on different hosts.

Verticillium dahliae is a soilborne vascular wilt fungus that is characterized by a broad host range of over 200 plant species (Fradin and Thomma 2006). Little is known about $V$. dahliae pathogenicity and, until recently, no effectors of this fungus had been characterized. Recently, the genome sequence of a $V$. dahliae strain has been determined (Klosterman et al. 2011) and, subsequently, the race 1-specific effector that is recognized by the Ve1 immune receptor of tomato was identified through comparative population genomics of race 1 and race 2 strains (de Jonge et al. 2012). This effector, named Ave1, is characterized as a small, secreted protein that is required for full virulence on tomato plants lacking the Vel resistance gene (de Jonge et al. 2012). Interestingly, Ave1 is homologous to a widespread family of plant natriuretic peptides, mobile signaling molecules that are secreted in the apoplast, particularly under conditions of biotic and abiotic stress, and play an important role in the regulation of water and ion homeostasis and, consequently, can affect many downstream processes, including photosynthesis (Gehring and Irvin 2003; Ruzvidzo et al. 2011). Ave1 homologs are found in a 
handful of plant-pathogenic fungi but a role in virulence has not yet been demonstrated for these homologs (de Jonge et al. 2012).

Query of the V. dahliae genome revealed that 127 (conserved) hypothetical proteins can be designated as small, cysteine-rich candidate effector proteins. However, no orthologs were found of the well-characterized SIX effectors that have been identified in F. oxysporum f. sp. lycopersici which, similar to $V$. dahliae, is also a vascular wilt pathogen of tomato (Takken and Rep 2010). Orthologs were only found of effectors that have previously been assigned to large superfamilies, such as the Hce2 family (homologs of Cladosporium fulvum effector Ecp2), the LysM effector family (homologs of C. fulvum effector Ecp6), and the NLP family (de Jonge and Thomma 2009; de Jonge et al. 2010; Klosterman et al. 2011; Stergiopoulos et al. 2012).

Years ago, the first $V$. dahliae NLP, named VdNEP, was already identified based on sequencing of expressed sequence tags (ESTs) from cultured mycelium of a cotton-pathogenic $V$. dahliae strain (Wang et al. 2004). The protein was found to induce typical defense responses, including cell death, in various plant species. Although a contribution to fungal aggressiveness was not demonstrated, it was suggested that VdNEP acts a wilt-inducing virulence factor (Wang et al. 2004). Interestingly, compared with other ascomycete plant pathogens, the $N L P$ gene family is expanded in the $V$. dahliae genome (Klosterman et al. 2011). Whereas most fungal genomes contain up to three NLP genes (Dallal et al. 2010; Garcia et al. 2007; Motteram et al. 2009; Schouten et al. 2008; Staats et al. 2007), $V$. dahliae was found to have eight NLP gene homologs (Klosterman et al. 2011). It has been speculated that the expansion of the NLP family, similarly reported for the genome of $F$. oxysporum, may contribute to the broad host range among dicotyledonous plant hosts (Klosterman et al. 2011; Ma et al. 2010). In this article, we describe our study of the NLP family of $V$. dahliae. We show that not all of the $V$. dahliae NLP display cytotoxic activity, and investigate the role of the cytotoxic
NLP in fungal virulence. Finally, we provide evidence for functional diversification not only with the $V$. dahliae NLP family but even between the cytotoxic NLP.

\section{RESULTS}

\section{Identification of the NLP family in tomato-pathogenic strain JR2.}

Recently, the NLP family of the defoliating cotton-pathogenic isolate V592 was reported to contain nine members (Zhou et al. 2012), while it was previously reported that the genome of the lettuce-pathogenic $V$. dahliae VdLs. 17 contains eight $N L P$ gene homologs (Klosterman et al. 2011). The difference in $N L P$ gene family size is due to the fact that Zhou and associates (2012) annotate gene VDAG_02984 as an NLP family member, whereas this member was not annotated as an NLP gene by Klosterman and associates (2011). To reevaluate the composition of the NLP family, an HMMER alignment search was performed on the proteome of the $V$. dahliae VdLs. 17 with the hidden Markov model (HMM) profile of the NLP-specific NPP1 domain (PF05630). Manual selection resulted in the identification of eight NLP family members, NLP1 to NLP7, and NLP9 (Table 1; Supplementary Figs. S1 and S2). A negative bit score for NLP8 disqualified this protein as a bona fide NLP family member due to insufficient support for the validity of the NPP1 domain, although the $E$ value suggests that the sequence is significantly related (Table 1). The degree of homology between the eight NLP members of $V$. dahliae VdLs. 17 is rather low (maximum $46.6 \%$ identity), and four of the NLP (NLP1, NLP2, NLP3, and NLP6) belong to type I containing two conserved Cys residues, whereas the remaining four (NLP4, NLP5, NLP7, and NLP9) belong to type II that contains four conserved Cys residues. Only NLP1 to NLP5 have an intact heptapeptide "GHRHDWE" motif whereas, in the remaining NLP, the first amino acid of this motif is changed from Gly to Ala (NLP6 and NLP7) or Asn (NLP9), and the sixth amino acid is changed from Trp into Phe (NLP6).

Table 1. Composition of the necrosis- and ethylene-inducing-like protein $(N L P)$ gene family in Verticillium dahliae

\begin{tabular}{|c|c|c|c|c|c|c|c|c|c|c|c|c|c|c|c|c|}
\hline \multirow[b]{2}{*}{ 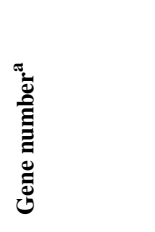 } & \multirow[b]{2}{*}{ 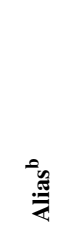 } & \multirow[b]{2}{*}{ } & \multirow[b]{2}{*}{ 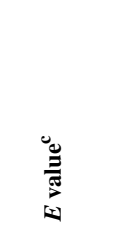 } & \multicolumn{11}{|c|}{ V. dahliae } & \multirow{2}{*}{$\begin{array}{c}\text { V. albo-atrum } \\
\end{array}$} & \multirow{2}{*}{ 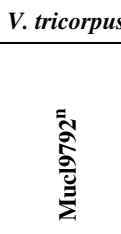 } \\
\hline & & & & $\begin{array}{l}\overrightarrow{1} \\
\overrightarrow{0} \\
\overrightarrow{0} \\
\vec{b}\end{array}$ & 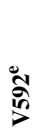 & $\stackrel{\widetilde{\Xi}}{\leftrightarrows}$ & 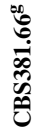 & 亘 & $\begin{array}{l}\bar{\sigma} \\
\overline{\dot{J}}\end{array}$ & 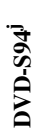 & 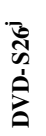 & 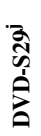 & $\begin{array}{l}\frac{4}{a} \\
\text { 官 }\end{array}$ & $\frac{\overline{6}}{\frac{1}{2}}$ & & \\
\hline VDAG_04701 & NLP1 & 23.9 & $9.70 \mathrm{E}^{-11}$ & + & + & + & + & + & + & + & + & + & + & + & + & - \\
\hline VDAG_01995 & NLP2 & 338.9 & $3.80 \mathrm{E}^{-98}$ & + & + & + & + & + & + & + & + & + & + & + & + & - \\
\hline VDAG_06993 & NLP3 & 382.4 & $2.90 \mathrm{E}^{-111}$ & + & + & + & + & + & + & + & + & + & + & + & + & + \\
\hline VDAG_04550 & NLP4 & 161.4 & $9.80 \mathrm{E}^{-45}$ & + & + & + & + & + & + & + & + & + & + & + & + & + \\
\hline VDAG_09117 & NLP5 & 107.1 & $2.30 \mathrm{E}^{-28}$ & + & + & + & + & + & + & + & + & + & + & + & + & - \\
\hline VDAG_04834 & NLP6 & 52.7 & $1.20 \mathrm{E}^{-12}$ & + & + & - & - & - & - & - & - & - & - & - & - & - \\
\hline VDAG_03497 & NLP7 & 113.1 & $3.30 \mathrm{E}^{-30}$ & + & + & + & + & + & + & + & + & + & + & + & + & + \\
\hline VDAG_02984 & NLP8 & -9 & $1.40 \mathrm{E}^{-08}$ & + & + & + & + & + & + & + & + & + & + & + & - & + \\
\hline VDAG_08022 & NLP9 & 64.9 & $1.10 \mathrm{E}^{-15}$ & + & + & + & + & + & + & + & + & + & + & + & + & - \\
\hline
\end{tabular}

\footnotetext{
${ }^{\mathrm{a}}$ Gene numbers according to the reference sequence of $V$. dahliae VdLs. 17 (Klosterman et al. 2011).

${ }^{\mathrm{b}}$ Naming according to Zhou and associates (2012).

${ }^{\mathrm{c}}$ Based on HMMER alignment search on $V$. dahliae VdLs.17 proteome with NLP-specific NPP1 domain (PF05630).

${ }^{\mathrm{d}}$ Lettuce-pathogenic isolate (Bhat and Subbarao, 1996).

${ }^{\mathrm{e}}$ Defoliating cotton-pathogenic isolate (Zhou et al. 2012).

${ }^{\mathrm{f}}$ Tomato-pathogenic isolate (Fradin et al. 2009).

${ }^{\mathrm{g}}$ Tomato-pathogenic isolate (Center for Biological Sequence Analysis, Utrecht, The Netherlands).

${ }^{\text {h }}$ Potato-pathogenic isolate.

${ }^{\mathrm{i}}$ Pistachio-pathogenic isolate.

j Soil isolate.

${ }^{\mathrm{k}}$ Tomato-pathogenic isolate.

${ }^{1}$ Potato-pathogenic isolate.

${ }^{\mathrm{m}}$ Alfalfa-pathogenic isolate (Bhat and Subbarao 1999).

${ }^{\mathrm{n}}$ Tomato-pathogenic isolate (Lievens et al. 2003).
} 
We subsequently queried the genome sequence of the tomatopathogenic strain JR2 (de Jonge et al. 2012) for its $N L P$ gene homologs. This analysis revealed seven $N L P$ genes, designated $N L P 1$ to NLP5, NLP7, and NLP9 (Table 1), whereas a homo$\log$ of NLP6 that is identified in strain VdLs.17 is not present in strain JR2. To further investigate the distribution of $N L P$ gene family members in the Verticillium genus, we queried the genomes of nine additional $V$. dahliae strains (de Jonge et al. 2012), as well as a $V$. tricorpus strain and a V. albo-atrum VaMs.102 (Klosterman et al. 2011). To this end, the DNA sequences of the $V$. dahliae strains were mapped to the $V$. dahliae VdLs.17 reference genome. The mapping analysis showed that the genomic regions harboring NLP1 to NLP5 and NLP7 to $N L P 9$ are covered by DNA sequences from the other $V$. dahliae strains, indicating the presence of these genes. However, similar to the JR2 strain, the genomic region harboring NLP6 was not found in any of the other $V$. dahliae strains. NLP6 was similarly lacking in $V$. albo-atrum VaMs.102 (Klosterman et al. 2011), while NLP8 is also lacking in this strain (Table 1). Interestingly, the $V$. tricorpus genome contains homologs of $N L P 3$, $N L P 4, N L P 7$, and NLP8 only.

Only NLP1 and NLP2 display cytotoxic activity.

Various reports have shown that NLP trigger necrosis upon infiltration into leaves of dicotyledenous plants. To test the necrosis-inducing activity of the NLP encoded by the tomatopathogenic JR2 strain, expression of the corresponding cDNAs in $N$. benthamiana by agroinfiltration was pursued. To this end, the coding sequences of the $N L P$ genes were cloned into the Gateway-compatible vector $p F A S T-R 02$ (Shimada et al. 2010) to generate expression constructs driven by the CaMV $35 \mathrm{~S}$ promoter, and transformed into Agrobacterium tumefaciens GV3101. Subsequently, the transgenic A. tumefaciens strains were infiltrated into the leaves of Nicotiana benthamiana plants. Leaf tissue started to collapse at 1 to 2 days post agroinfiltration of $N L P 1$ and $N L P 2$, and the infiltrated leaves developed clear necrosis by 3 days post agroinfiltration (Fig. 1A). In contrast, no tissue collapse or necrosis could be observed upon agroinfiltration of any of the other $N L P$ genes. Western analysis performed on total protein extracts of the

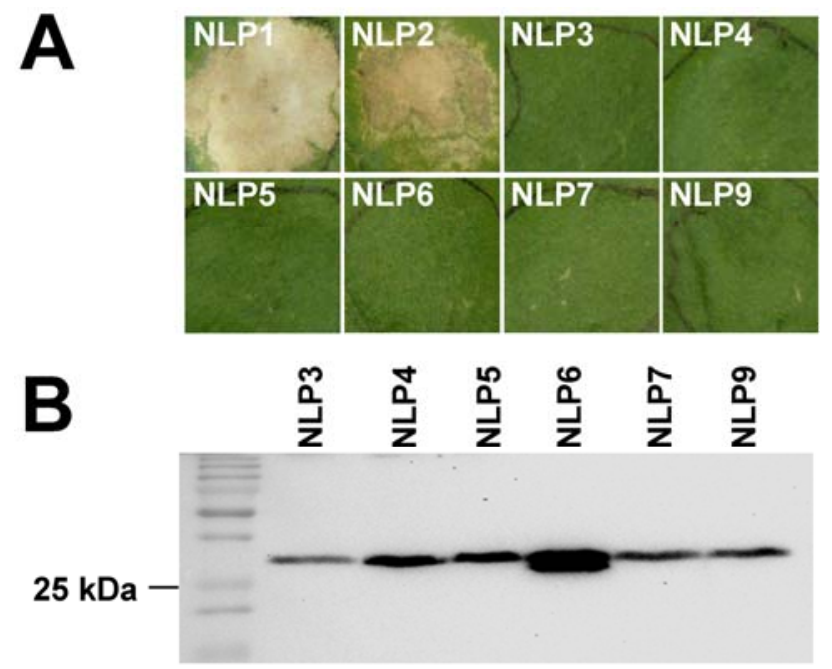

Fig. 1. Only expression of Verticillium dahliae necrosis- and ethyleneinducing-like protein $(N L P) 1$ and NLP2 induces cell death in Nicotiana benthamiana. A, Agroinfiltration of constructs for constitutive expression of $V$. dahliae NLP genes reveals necrosis at the injection sites for NLP1 and $N L P 2$ but none of the other NLP genes. Pictures were taken at 3 days after agroinfiltration. B, Western blot analysis on total protein extracts of $N$. benthamiana upon agroinfiltration of constructs for constitutive expression of $V$. dahliae NLP genes that did not induce necrosis in A. agroinfiltrated leaf area confirmed the presence of the proteins that did not induce necrosis in planta (Fig. 1B).

To confirm the differential cytotoxic activity, the NLP were also produced in the yeast Pichia pastoris and purified. Whereas production of NLP6 and NLP9 failed, sufficient amounts of protein were obtained from the other NLP and infiltrated abaxially into leaves of $N$. tabacum plants. Also in this experiment, only infiltration of NLP1 and NLP2 resulted in tissue necrosis, whereas infiltration of NLP3 to NLP5 and NLP7 did not trigger necrosis (Supplementary Fig. S3). Collectively, these data show that only NLP1 and NLP2 display cytotoxic activity, whereas the remaining $V$. dahliae NLP do not.

\section{$N L P 1$ and $N L P 2$ are expressed}

in planta but not under control of VdSge1.

It has previously been shown for one of the cytotoxic NLP from $V$. dahliae that it has the potential to induce necrosis and wilting in planta; however, a role in virulence on plant hosts has not been demonstrated thus far for any of its cytotoxic NLP (Wang et al. 2004). To address such a role, it was assessed whether both cytotoxic NLP are expressed during host colonization. To this end, $V$. dahliae JR2 was root inoculated on susceptible plants of 'MoneyMaker' tomato, and complete plants were harvested at regular intervals up to 16 days postinoculation (dpi). After RNA isolation and cDNA synthesis, real-time polymerase chain reaction (PCR) was performed to assess $N L P 1$ and NLP2 transcript levels that were calibrated using transcript levels of the elongation factor $1-\alpha$ gene as a refer-
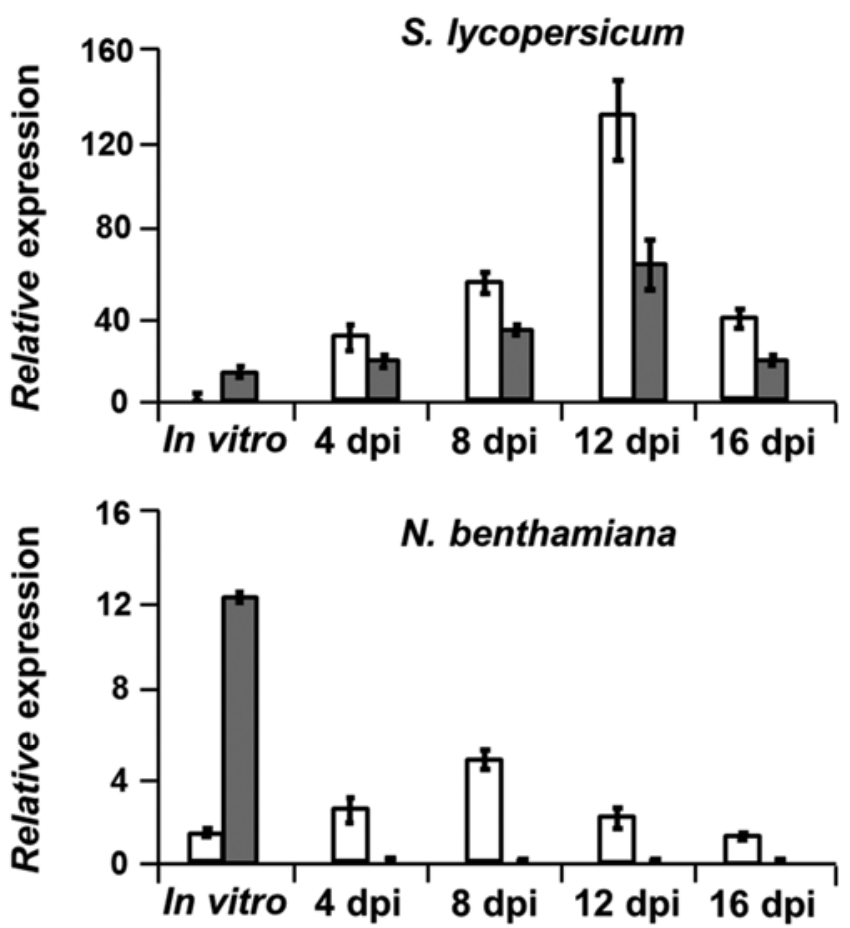

Fig. 2. Necrosis- and ethylene-inducing-like protein $(N L P) 1$ and $N L P 2$ expression during infection of Verticillium dahliae on tomato and Nicotiana benthamiana. Plants of 10-day-old tomato (Solanum lycopersicum) 'MoneyMaker' (top graph) or 15-day-old $N$. benthamiana (bottom graph), were root inoculated with wild-type $V$. dahliae and harvested at regular intervals from 4 to 16 days postinoculation (dpi). After RNA isolation and cDNA synthesis, real-time polymerase chain reaction was performed to determine the relative expression levels of $N L P 1$ (white bars) and $N L P 2$ (gray bars) using the $V$. dahliae elongation factor $1-\alpha$ gene as a reference, and compared with expression upon $V$. dahliae growth in vitro in $\mathrm{Mu}$ rashige-Skoog medium. NLP1 and NLP2 expression levels are compared with expression of $N L P 1$ in vitro, which is set to 1 . No $N L P 2$ expression was detected in $N$. benthamiana. 
ence. This analysis demonstrated that both $N L P 1$ and $N L P 2$ are induced in planta, with a similar expression pattern that peaks approximately $12 \mathrm{dpi} \mathrm{(Fig.} \mathrm{2).} \mathrm{By} \mathrm{this} \mathrm{point} \mathrm{in} \mathrm{time,} \mathrm{major}$ symptoms of disease, including the onset of necrosis and significant stunting, become apparent in susceptible tomato plants.

We recently demonstrated that V. dahliae Sgel (VdSgel) is required for growth and development as well as for pathogenicity on tomato (Santhanam and Thomma 2013). In contrast to $F$. oxysporum Sge1, we found that $\mathrm{VdSge} 1$ is not a general regulator of effector gene expression, because some candidate effector genes were negatively regulated by VdSge1, some positively, and others not (Santhanam and Thomma 2013). Because the VdSgel deletion mutant is not pathogenic on tomato, assessment of NLP1 and NLP2 gene expression was assessed in a tomato cell suspension culture (Santhanam and Thomma 2013). To this end, a suspension of MSK8 tomato cells was inoculated with conidia of a VdSgel deletion strain and the cor-

A

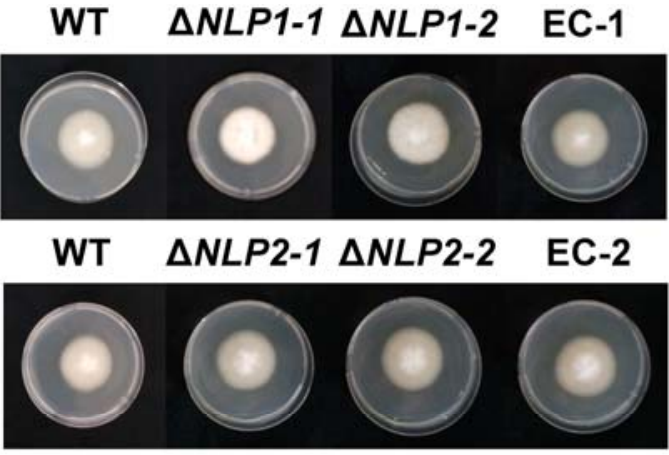

B

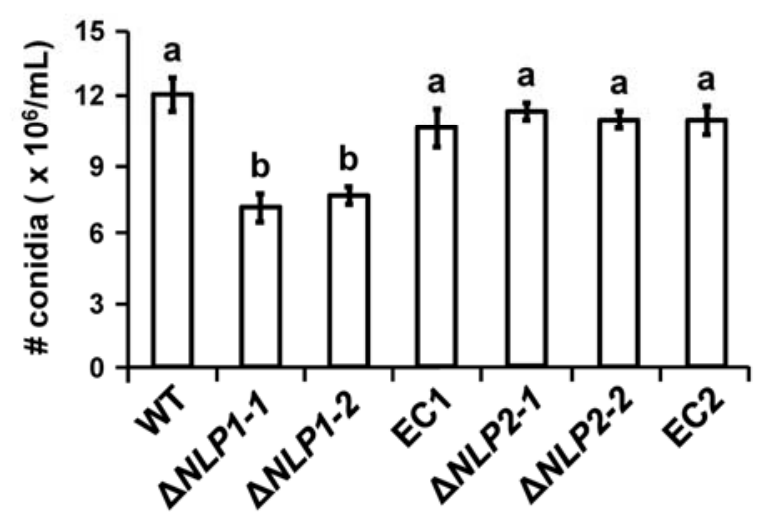

C
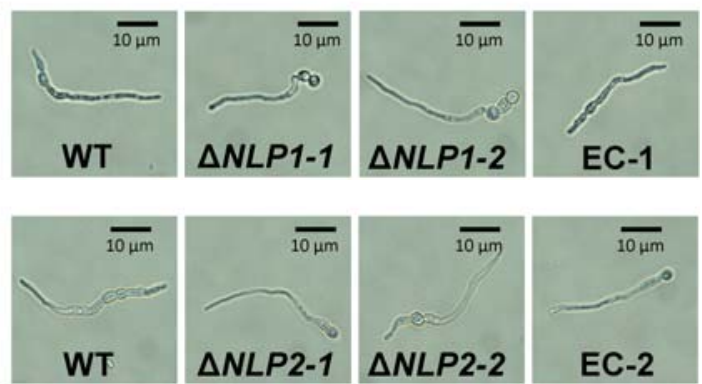

Fig. 3. Targeted deletion of necrosis- and ethylene-inducing-like protein $(N L P) 1$ affects conidiospore production. A, Radial growth and colony morphology of wild-type Verticillium dahliae (WT), two NLP1 deletion strains ( $\triangle N L P 1-1$ and $\triangle N L P 1-2)$, two NLP2 deletion strains ( $\triangle N L P 2-1$ and $\triangle N L P 2-2)$, and ectopic transformants (EC1 and EC2) after 7 days of incubation on PDA medium at $22^{\circ} \mathrm{C}$. B, Average number of conidia produced after 7 days of growth on potato dextrose agar medium based on two independent experiments. Different letters indicate significant differences at $P<$ 0.05 as calculated with Student's $t$ test. C, Microscopic observation of the germination of conidia at $16 \mathrm{~h}$ after incubation in Czapek-Dox medium. responding wild type and, after $96 \mathrm{~h}$, the cells were harvested and examined for $N L P 1$ and $N L P 2$ expression with real-time PCR. Expression of $N L P 1$ and $N L P 2$ was not abolished in the $V d S g e 1$ deletion strain; therefore, we conclude that the expression of the genes encoding cytotoxic NLP is not governed by VdSge1 (Supplementary Fig. S4).

\section{NLP1 affects vegetative growth and conidiospore production.}

Because both NLP1 and NLP2 were found to be expressed in $V$. dahliae during colonization of tomato plants, targeted deletion was pursued by replacement of the coding sequence of each of the genes by a hygromycin resistance cassette through homologous recombination. Deletion of the coding sequences was subsequently verified by PCR. Several independent deletion mutants were obtained for each of the genes, of which two were used for further analysis in this study (Supplementary Fig. S5). To assess the role of the cytotoxic NLP on growth and development, various growth characteristics were assessed. Radial growth of the NLP1 and NLP2 deletion strains was similar to that of the wild-type strain and of the ectopic transformants (Fig. $3 \mathrm{~A})$. However, when conidiospores were isolated from 1-weekold cultures, $N L P 1$ deletion strains appeared to have developed approximately $40 \%$ less conidiospores than NLP2 deletion strains, the wild-type strain, and ectopic transformants (Fig. 3B). Nevertheless, germination of the conidiospores was similar for all strains, including the $N L P 1$ deletion strains (Fig. 3C). The reduced conidiospore production of the $N L P 1$ deletion strains

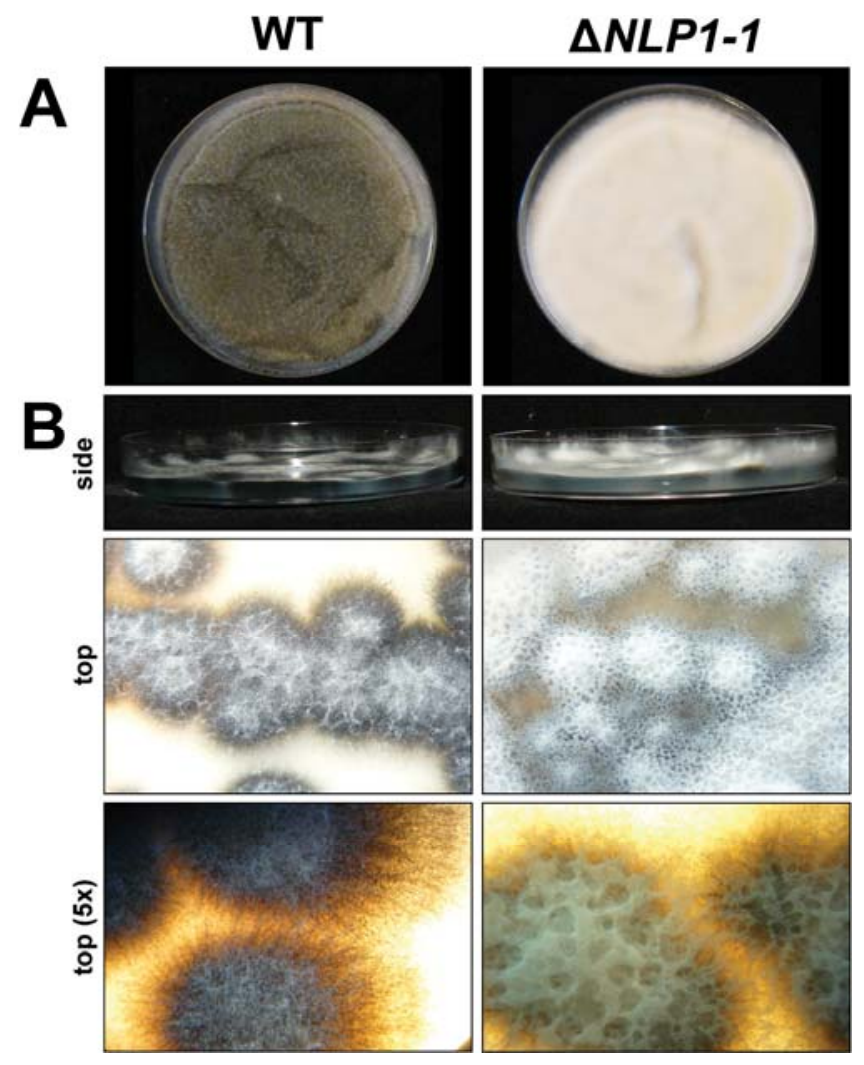

Fig. 4. Targeted deletion of necrosis- and ethylene-inducing-like protein $(N L P) 1$ induces formation of aerial hyphae. A, Morphological appearance of wild-type Verticillium dahliae (WT) and an NLP1 deletion strain ( $\triangle N L P 1-1)$ after 14 days of incubation on potato dextrose agar (PDA) medium at $22^{\circ} \mathrm{C}$. B, WT $V$. dahliae and an NLP1 deletion strain ( $\left.\triangle N L P 1-1\right)$ after 21 days of incubation on PDA medium at $22^{\circ} \mathrm{C}$. Top pictures show side views of petri dishes with fungal strains on PDA medium, showing aerial hyphae for the $N L P I$ deletion strain. Middle and lower pictures show magnification of fungal colonies. 
was overcome by complementation with a genomic construct containing the wild-type NLPl gene, demonstrating that the reduced conidiospore production is a genuine phenotype of NLP1 deletion (Supplementary Fig. S6B). When the fungal cultures got older, NLP1 deletion strains became morphologically distinct. Whereas the color of the wild-type strain, NLP2 deletion strains, and ectopic transformants became gray, $N L P 1$ deletion strains were white (Fig. 4A). Closer inspection revealed that the white appearance was due to extensive formation of aerial hyphae that covered the fungal colonies of the NLP1 deletion strains on the potato dextrose agar (PDA) plates (Fig. 4B). Also, this phenotype was overcome by complementation of the deletion strain with a genomic construct encoding the wild-type NLP1 gene. All other fungal strains did not show the extensive formation of these aerial hyphae (Supplementary Fig. S7). In support for a role in absence of host colonization during growth, we found that NLP are expressed in vitro, because 228 reads belonging to this $N L P$ gene family member were retrieved in the RNAseq data of $V$. dahliae grown in Czapek-Dox medium (de Jonge et al. 2012). Collectively, these data support a role for $N L P 1$ in vegetative growth and asexual reproduction.

\section{$N L P 1$ and $N L P 2$ are required for virulence.}

To assess the role of $N L P 1$ and $N L P 2$ in pathogenicity on tomato plants, the deletion strains were root inoculated on sus-
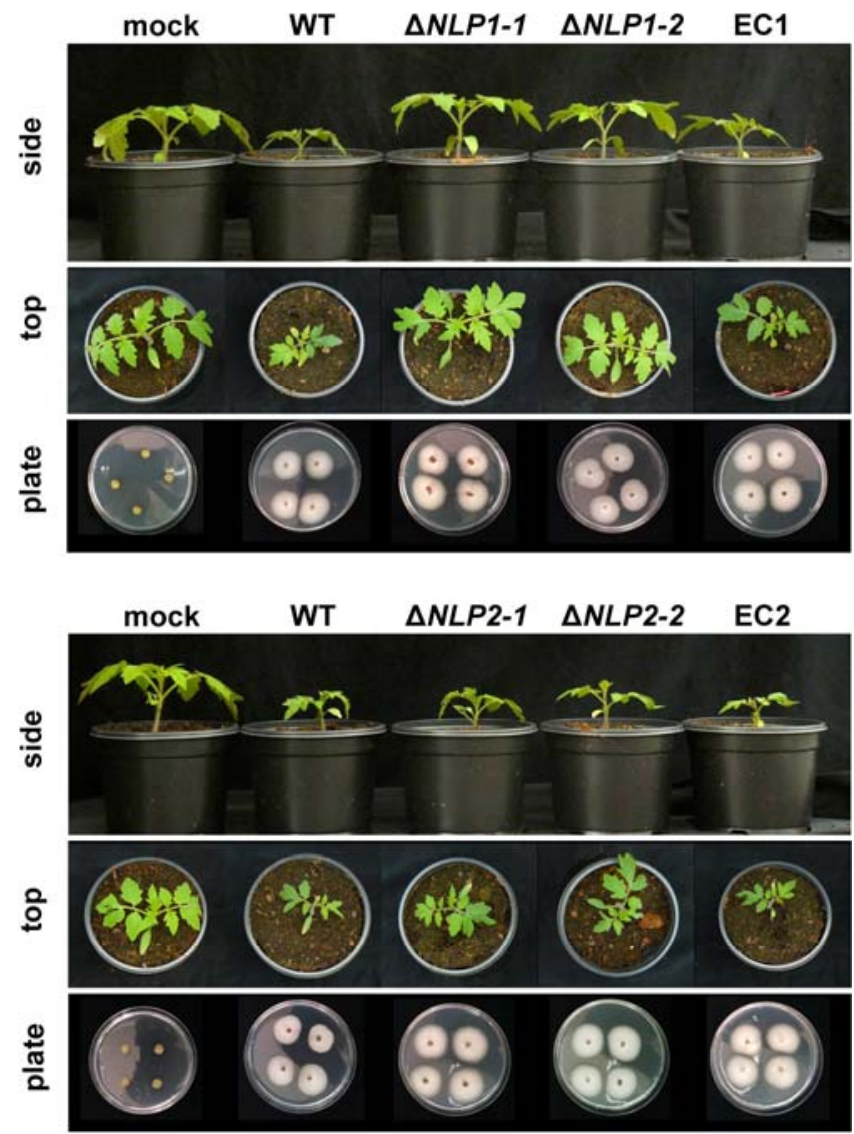

Fig. 5. Necrosis- and ethylene-inducing-like protein $(N L P) 1$ and $N L P 2$ are required for virulence of Verticillium dahliae on tomato. Pathogenicity assay to investigate the role of NLP1 (top panel) and NLP2 (bottom panel) in $V$. dahliae virulence. Side and top view of 'MoneyMaker' tomato plants that were mock inoculated (mock) or inoculated with wild-type $V$. dahliae (WT), two $N L P$ deletion strains ( $\triangle N L P 1-1$ and $\triangle N L P 1-2)$, two $N L P 2$ deletion strains ( $\triangle N L P 2-1$ and $\triangle N L P 2-2)$, and corresponding ectopic transformants (EC1 and EC2) at 14 days postinoculation are shown. Fungal outgrowth at 7 days after plating of stem sections harvested at 14 days postinoculation are shown at the bottom of each panel. ceptible MoneyMaker tomato plants along with the wild-type strain and ectopic transformants. Interestingly, whereas ectopic transformants were found to be as virulent as the wild-type strain, $N L P 1$ as well as $N L P 2$ deletion strains were found to be significantly less pathogenic (Fig. 5). The plants that were inoculated with the $N L P 1$ and NLP2 deletion strains still developed Verticillium wilt symptoms but were less stunted and showed less typical disease symptoms (e.g., darkening of the leaves) than plants that were inoculated with ectopic transformants or the wild-type strain (Fig. 5). Intriguingly, deletion of $N L P 1$ seemed to have a stronger effect on virulence than deletion of $N L P 2$, because plants inoculated with $N L P 1$ deletion strains remained taller and showed fewer symptoms of infection than plants inoculated with NLP2 deletion strains (Fig. 5). This was confirmed upon measuring the surface area of the foliage of the plants inoculated with the various fungal genotypes, because plants that were inoculated with NLP1 deletion strains developed as much foliage as mock-inoculated plants, whereas the amount of foliage developed by plants that were inoculated with $N L P 2$ deletion strains was significantly reduced (Fig. 6A). Nevertheless, plants that were inoculated with NLP2 deletion strains developed significantly more foliage than those inoculated with either the wild-type strain or ectopic transformants (Fig. 6A). Fungal outgrowth assays upon plating of stem sections harvested from the hypocotyls of the inoculated plants demonstrated that $N L P 1$ and $N L P 2$ deletion strains were still able to colonize tomato plants (Fig. 5). However, real-time PCR quantification of fungal biomass demonstrated

A
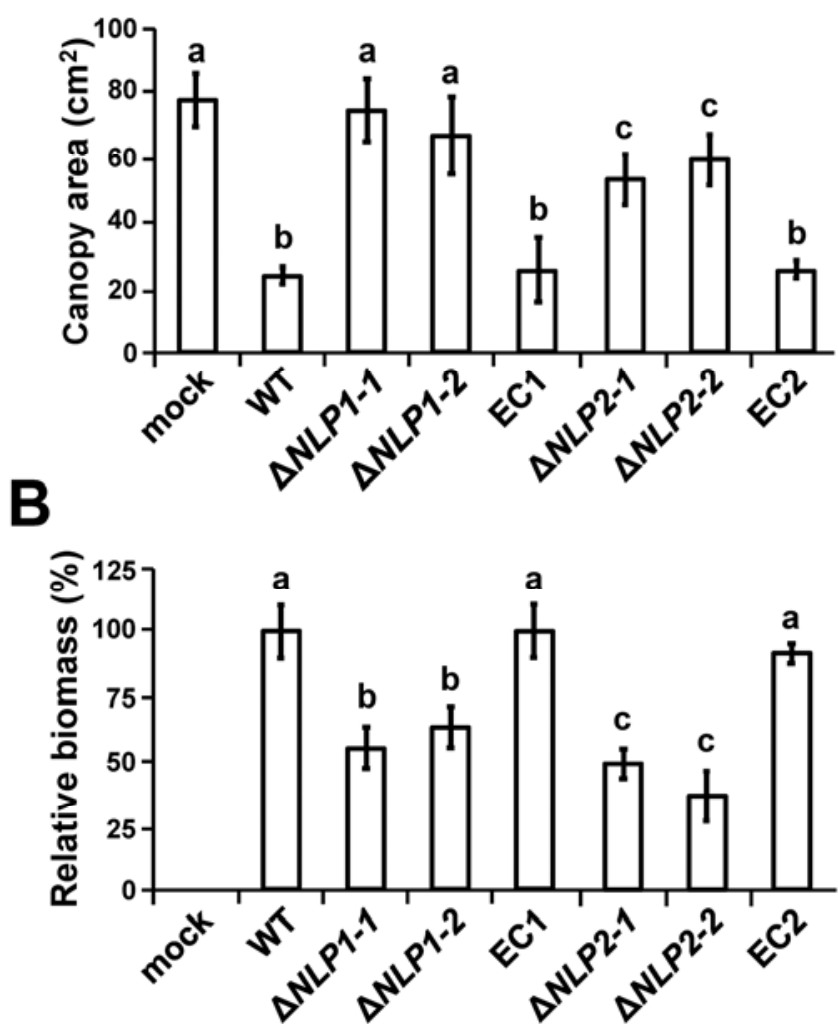

Fig. 6. Necrosis- and ethylene-inducing-like protein $(N L P) 1$ and $N L P 2$ are required for virulence of Verticillium dahliae on tomato. A, Average canopy area of 6 'MoneyMaker' tomato plants at 14 days after mock inoculation (mock) or inoculation with wild-type $V$. dahliae (WT), two NLP1 deletion strains ( $\triangle N L P 1-1$ and $\triangle N L P 1-2)$, two $N L P 2$ deletion strains ( $\triangle N L P 2-1$ and $\triangle N L P 2-2)$, and corresponding ectopic transformants (EC1 and EC2). B, Real-time polymerase chain reaction quantification of fungal biomass. Different letter labels indicate significant differences $(P<0.05)$. 
that NLP1 and NLP deletion strains developed significantly less fungal biomass in planta than the ectopic transformants and the wild-type strain (Fig. 6B).

To investigate whether the observed role of NLP1 and NLP2 in pathogenicity is confined only to tomato or also concerns other host species, we tested the pathogenicity of the NLP1 and NLP2 deletion strains on the Brassicaceous and Solanaceous model plants Arabidopsis thaliana and N. benthamiana, respectively. Similar to tomato, targeted deletion of NLP1 as well as of NLP2 resulted in markedly compromised virulence on Arabidopsis plants, because they were clearly less stunted (Fig. 7A). In contrast, a differential contribution of $N L P 1$ and $N L P 2$ to $V$. dahliae virulence was monitored on $N$. benthamiana. Whereas targeted deletion of NLPl resulted in markedly compromised virulence on $N$. benthamiana, targeted deletion of NLP2 did not result in significantly compromised virulence on this host plant (Fig. 7B). This was confirmed not only by measurement of the surface area of the foliage of the plants inoculated with the various fungal genotypes but also by realtime PCR quantification of fungal biomass (Fig. 8). Considering the observation that NLP2 displays cytotoxic activity also on $N$. benthamiana (Fig. 1), the observation that NLP2 deletion did not result in significantly compromised virulence on this host plant prompted the question of whether this gene is expressed during $N$. benthamiana colonization. Surprisingly, whereas $N L P 1$ is expressed during colonization of $N$. benthamiana by $V$. dahliae in a pattern similar to that observed on tomato (Fig. 2), no expression of NLP2 could be detected at all

A
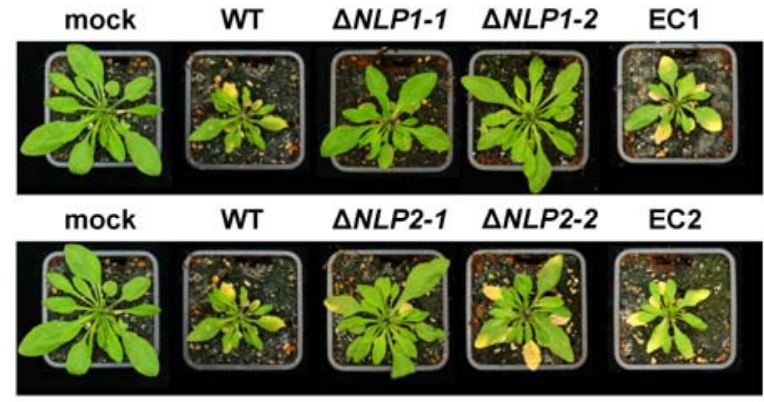

B
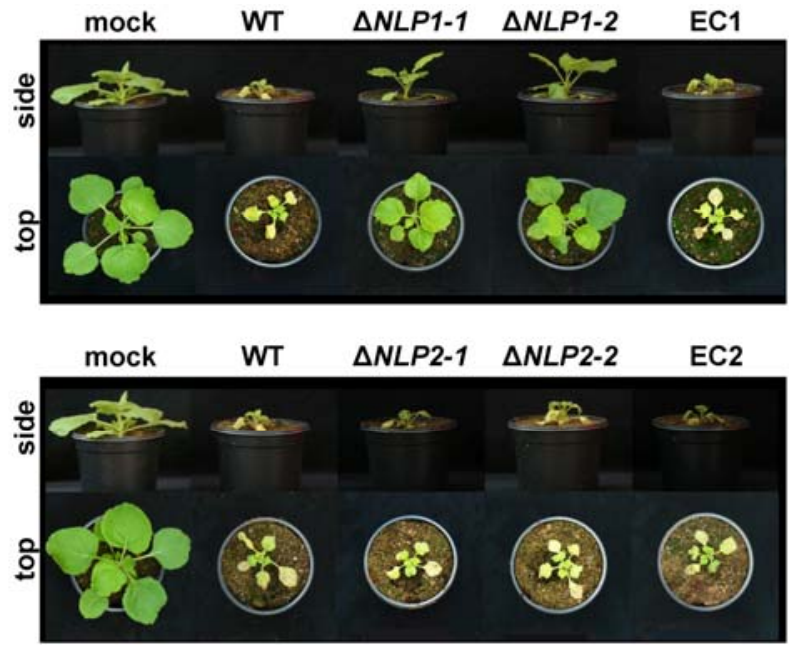

Fig. 7. Requirement of necrosis- and ethylene-inducing-like protein $(N L P) 1$ and $N L P 2$ for virulence of Verticillium dahliae on Arabidopsis and Nicotiana benthamiana. A, Top view of Arabidopsis plants of the Col-0 ecotype and $\mathbf{B}$, top and side view of $N$. benthamiana plants that were mock inoculated (mock) or inoculated with wild-type $V$. dahliae (WT), two $N L P 1$ deletion strains ( $\triangle N L P 1-1$ and $\triangle N L P 1-2)$, two $N L P 2$ deletion strains ( $\triangle N L P 2-1$ and $\triangle N L P 2-2)$, and corresponding ectopic transformants (EC1 and EC2) at 21 days postinoculation (dpi) (Arabidopsis) and 14 dpi ( $N$. benthamiana) are shown. in this plant species. Nevertheless, overall, it can be concluded that NLP1 and NLP2 are required for virulence on various host plants.

\section{DISCUSSION}

In this study, we provide evidence for significant functional diversification within the NLP family of a fungal pathogen. We have shown that targeted deletion from the $V$. dahliae genome of $N L P 1$, encoding a cytotoxic NLP that contributes to virulence on various host plants, directly affects growth and conidiospore production in vitro. Because this finding was highly unexpected, we carefully checked the veracity of our findings. To generate the NLPI deletion construct, genomic sequences flanking the NLPl coding sequence were used; more specifically, a 1.4-kb upstream and 250-bp downstream sequence. Approximately $2.2 \mathrm{~kb}$ upstream of $N L P 1$ lies a gene encoding a vegetative cell wall protein (VDAG_04700). Although this gene lies well outside the range of sequence that was used to generate the construct for targeted deletion of NLP1 by homologous recombination, we confirmed that expression of this gene was not affected in the NLPl deletion strains when compared with the wild-type $V$. dahliae upon growth in vitro. No genes other than $N L P 1$ were annotated in the region that was used for homologous recombination. Furthermore, mapping of sequence reads obtained with RNAseq from a time course of $V$. dahlia-infected $N$. benthamiana resulted in the identification of reads for over 8,000 V. dahliae genes (de Jonge et al. 2012) but no reads were mapped in the range of sequence that was used to generate the construct for homologous recombination other than those belonging to NLP1. Furthermore, the in vitro

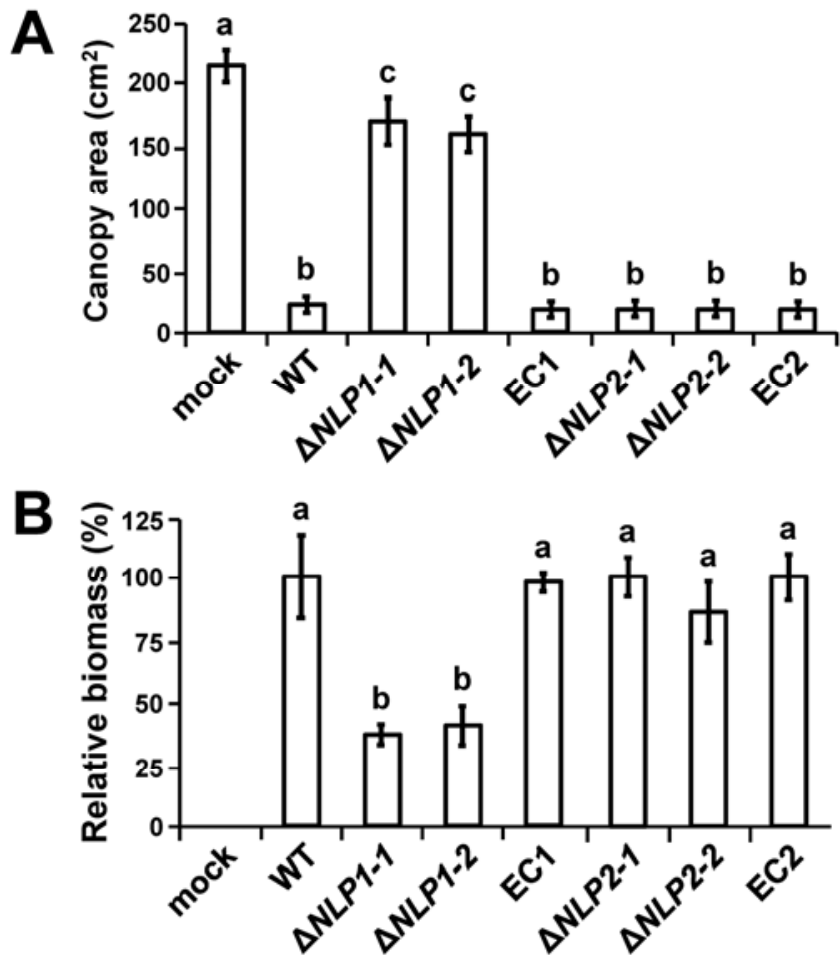

Fig. 8. Necrosis- and ethylene-inducing-like protein $(N L P) 1$ but not $N L P 2$ is required for virulence of Verticillium dahliae on Nicotiana benthamiana. A, Average canopy area of six $N$. benthamiana plants at 14 days after mock inoculation (mock) or inoculation with wild-type $V$. dahliae (WT), two $N L P 1$ deletion strains ( $\triangle N L P 1-1$ and $\triangle N L P 1-2)$, two $N L P 2$ deletion strains $(\triangle N L P 2-1$ and $\triangle N L P 2-2)$, and corresponding ectopic transformants (EC1 and EC2). B, Real-time polymerase chain reaction quantification of fungal biomass. Different letter labels indicate significant differences $(P<0.05)$. 
growth and conidiation phenotype was overcome by complementation of the NLP1 deletion strain with a genomic construct containing the wild-type $N L P 1$ gene. Finally, coinciding with a role in vitro in the absence of the host, our data show that NLP1 is expressed in $V$. dahliae during growth in vitro. Thus, we conclude that $N L P 1$ unambiguously affects in vitro growth and conidiation and, with this observation, a novel function for a cytotoxic NLP outside the host plant is revealed.

The observed role for a cytotoxic NLP outside the plant and pathogen virulence was unexpected, and obviously leads to the question of how such a secreted effector protein affects growth and conidiation. In this respect, it is interesting to note that analysis of the crystal structure of a cytotoxic NLP from the phytopathogenic oomycete Pythium aphanidermatum revealed structural homology not only to actinoporins, a family of poreforming toxins produced by sea anemones, but also to particular fungal lectins (Ottmann et al. 2009). All these proteins are small polypeptides containing a central $\beta$-sandwich architecture surrounded by helices, and both the actinoporins and lectins are soluble proteins that target cellular surfaces via a surfaceexposed cavity (Birck et al. 2004; Ottmann et al. 2009). One of these lectins, XCL from Xerocomus chrysenteron, is an insecticidal protein that binds to $\mathrm{N}$-acetyl galactosamine at the cell surface of target cells, after which it is internalized and changes the actin cytoskeleton (Birck et al. 2004). It is tempting to speculate that NLP1 will bind to particular fungal cell wall carbohydrates, after which internalization occurs to influence developmental processes.

Further evidence for functional diversification within the $V$. dahliae NLP family comes from the identification of noncytotoxic family members. Most fungal genomes contain up to three NLP genes (Dallal et al. 2010; Garcia et al. 2007; Motteram et al. 2009; Schouten et al. 2008; Staats et al. 2007). Nevertheless, although the $V$. dahliae NLP family is significantly expanded, this fungus also contains only two genes that encode cytotoxic NLP. Possibly, the noncytotoxic NLP contribute to pathogen virulence through mechanisms other than inducing cellular leakage. However, our data for NLP1 suggest that diversification toward functions other than virulence may be likely as well. Thus far, noncytolytic NLP have only been described for oomycete species that are generally characterized by significantly expanded NLP families, containing up to 70 potential NLP genes (Dong et al. 2012). Whereas some oomycetes contain cytolytic and noncytolytic NLP (Dong et al. 2012; Kanneganti et al. 2006), others contain only noncytolytic NLP, such as the obligate biotrophic oomycete pathogen Hyaloperonospora arabidopsidis (Cabral et al. 2012). The finding that the noncytotoxic $H$. arabidopsidis NLP are expressed during early stages of infection has led to the hypothesis that these NLP still exert functions related to host colonization (Cabral et al. 2012). However, also for $H$. arabidopsidis NLP, alternative roles that are not related to virulence may occur as well. A role in growth and development outside the host plant may, at the same time, explain why $N L P$ genes also occur in nonpathogenic microorganisms.

Our study confirms the findings of recent study on a virulent cotton-infecting $V$. dahliae isolate, for which nine potential NLP were identified, of which two displayed cytotoxic activity (Zhou et al. 2012). However, in contrast to our findings, targeted deletion of the genes encoding the cytotoxic NLP did not affect $V$. dahliae virulence on cotton (Zhou et al. 2012). In our study, we found that targeted deletion of NLP1 as well as of $N L P 2$ significantly compromised $V$. dahliae virulence on tomato and Arabidopsis, whereas deletion of NLP1 but not of $N L P 2$ compromised virulence on $N$. benthamiana. However, the differential role of $N L P 2$ we observed in virulence on different hosts could be attributed to the observation that $N L P 2$ is not expressed during infection on $N$. benthamiana. This is not the case for the study on cotton because, despite the absence of a virulence phenotype for the corresponding deletion strains, $N L P 1$ as well as $N L P 2$ were found to be expressed during infection (Zhou et al. 2012). Moreover, targeted deletion of both genes simultaneously did not affect fungal virulence, suggesting that the role of the cytotoxic NLP in infection of cotton is dispensable and further supporting the differential contribution of cytotoxic NLP to virulence of $V$. dahliae. It is not surprising that a broad-host-range pathogen such as $V$. dahliae that is able to infect hundreds of hosts will express effectors that do not contribute to virulence on all of its hosts.

In conclusion, our data demonstrate extensive functional diversification within the expanded NLP family of the fungal plant pathogen $V$. dahliae. We have demonstrated differential cytotoxicity among the family members, a differential contribution to virulence of the cytotoxic family members, and, finally, also a differential effect of the cytotoxic family members outside the host plant on growth and conidiospore production. Future efforts will be devoted to investigate further functional diversification with the group of noncytotoxic $V$. dahliae NLP.

\section{MATERIALS AND METHODS}

Identification of Verticillium NLP family members.

The HMM profile of the NPP1 domain (PF05630) was retrieved from the Pfam database and an HMMER alignment search was performed against the $V$. dahliae VdLs.17 proteome using default parameters. The resulting hits were manually selected for positive bit scores and $E$ values $<1 \mathrm{e}-10$. Presence of signal peptides was confirmed using SignalP3.0 with default parameters. Homologous NLP genes were identified in other $V$. dahliae strains by mapping the Illumina DNA sequence reads of these strains (de Jonge et al 2012) with the gsnap/gmap software to the reference genome of $V$. dahliae VdLs.17 using default settings (Wu and Nacu 2010; Wu and Watanabe 2005). Similarly, NLP homologs were identified using Illumina DNA sequence reads of $V$. tricorpus MUCL9792 (L. Faino and B. P. H. J. Thomma, unpublished data), and reciprocal BLAST searches to the $V$. dahliae VdLs.17 genome were used to confirm the identities of the $N L P$ homologs.

\section{Phylogenetic analyses.}

Phylogenetic analyses of the $V$. dahliae NLP family was conducted using the Phylogeny.fr web-service. Sequences were aligned with MUSCLE and curated with Gblocks. The phylogenetic tree was reconstructed using the PhyML program (maximum likelihood method) and statistical reliability was tested using bootstrap with 500 replications.

\section{Fungal transformations.}

To generate $N L P 1$ and $N L P 2$ deletion constructs, flanking sequences of the NLPI and NLP2 coding sequences were amplified from genomic DNA of $V$. dahliae JR2 using the primers mentioned in Supplementary Table S1. The amplified products were cloned into pRF-HU2 as described previously (Frandsen et al 2008).

To generate the NLP1 complementation construct, a 1,871bp EcoRI/PacI fragment containing the NLPl coding sequence with a 998-bp upstream and 170-bp downstream sequence was amplified from $V$. dahliae JR2 genomic DNA, and cloned into binary vector pBT081 (Houterman et al 2008).

Agrobacterium tumefaciens-mediated transformation of $V$. dahliae was performed as described previously (Santhanam 2012), and transformants were selected on PDA supplemented with cefotaxime (Duchefa, Haarlem, The Netherlands) at 200 
$\mu \mathrm{g} / \mathrm{ml}$ and hygromycin (Duchefa) at $50 \mu \mathrm{g} / \mathrm{ml}$. Homologous recombination was verified by PCR. Complementation transformants were selected on PDA supplemented with cefotaxime at $200 \mu \mathrm{g} / \mathrm{ml}$ and phleomycin at $100 \mu \mathrm{g} / \mathrm{ml}$ (InvivoGen, San Diego, CA, U.S.A.).

\section{Pathogenicity assays.}

Pathogenicity assays were performed on 10-day-old tomato seedlings (MoneyMaker), 2-week-old Arabidopsis thaliana (Col-0), and 2-week-old $N$. benthamiana plants using root-dip inoculation as previously described (Fradin et al. 2009). Disease symptoms were scored up to $14 \mathrm{dpi}$ (tomato and $N$. benthamiana) or $21 \mathrm{dpi}$ (Arabidopsis), pictures were taken, and ImageJ was used to determine the canopy area. To determine in planta colonization, stem sections at the height of the first internode were taken, surface sterilized, sliced, placed on PDA supplemented with chloramphenicol at $50 \mu \mathrm{g} / \mathrm{ml}$, and incubated at $22^{\circ} \mathrm{C}$. Fungal outgrowth was monitored after 7 days. For in planta biomass quantification, roots of three inoculated plants were harvested at $14 \mathrm{dpi}$. The samples were ground to powder and genomic DNA was isolated. Real-time PCR on genomic DNA was carried out using an ABI7300 PCR machine (Applied Biosystems, Foster City, CA, U.S.A.) in combination with quantitative PCR core kit for SYBR Green I (Eurogentec, Seraing, Belgium) and analyzed using the 7300 SDS software (Applied Biosystems). Verticillium elongation factor 1- $\alpha$ was used to quantify fungal colonization. Tomato, Arabidopsis, and $N$. benthamiana actin primers were used as endogenous plant control.

\section{Growth, conidiogenesis, and germination assays.}

Radial growth was monitored by placing a $2-\mu 1$ droplet of a conidial suspension of $10^{6}$ conidia/ml in the center of a PDA plate and incubating it at $22^{\circ} \mathrm{C}$. For quantification of conidia production, $5 \mathrm{ml}$ of water was added to the culture and conidial suspensions were prepared. Ten-times diluted conidial suspensions were counted in a hemocytometer. To determine the germination of conidia, $10^{6}$ conidia $/ \mathrm{ml}$ were inoculated in Czapek-Dox medium at $22^{\circ} \mathrm{C}$ for $16 \mathrm{~h}$ and observed under a microscope.

\section{Cytotoxic activity determination.}

For transient expression of $N L P$ genes in $N$. benthamiana, the full-length coding sequences containing a $3^{\prime}$ FLAG tag were amplified and cloned into $\mathrm{pENTR/D-TOPO} \mathrm{(Invitrogen,}$ Carlsbad, CA, U.S.A.). After sequence verification, the inserts were subcloned in the destination vector pFAST-R02 (Shimanda et al 2010) and transformed to Agrobacterium tumefaciens GV3101 by electroporation. A. tumefaciens strains were subsequently grown in yeast extract broth at $28^{\circ} \mathrm{C}$ overnight. The overnight cultures were diluted to an optical density at $600 \mathrm{~nm}$ of 0.5 with MMA (10 mM MES, $10 \mathrm{mM} \mathrm{MgCl} 2,100 \mu \mathrm{M}$ acetosyringone, $\mathrm{pH}$ 5.7) containing acetosyringone at $1 \mathrm{ml} / \mathrm{liter}$ and incubated at room temperature for 3 to $4 \mathrm{~h}$. The cultures were infiltrated on the abaxial side of the youngest, fully expanded leaves of 4-week-old $N$. benthamiana.

To verify protein production, leaves without midveins were harvested at 3 dpi and flash frozen in liquid nitrogen. Total proteins were extracted using P-PER Plant Protein Extraction Kit (Pierce Biotechnology, Rockford, IL, U.S.A.) following the manufacturer's instructions. The proteins were separated using $15 \%$ sodium dodecyl sulfate polyacrylamide electrophoresis gels. Immunoblotting was performed using ANTI-FLAG M2 antibody and the proteins were visualized by enhanced chemiluminenscence (Pierce Biotechnology

NLP production in $P$. pastoris was performed essentially as described previously (Ottmann et al. 2009).
Gene expression analysis.

To determine the in planta expression of $N L P 1$ and $N L P 2$, 10-day-old tomato seedlings and 2-week-old $N$. benthamiana plantlets were root inoculated with $V$. dahliae. Whole plants were harvested at $4,8,12$, and $16 \mathrm{dpi}$ and flash frozen in liquid nitrogen. After grinding, $100 \mathrm{mg}$ of ground material was used for total RNA extraction (Qiagen, Venlo, The Netherlands) and cDNA synthesis (Invitrogen). Real-time PCR was performed to determine NLP1 and NLP2 expression. Verticillium elongation factor 1- $\alpha$ was used as an endogenous control and reactions were performed in triplicate. Real-time PCR conditions consisted of an initial $95^{\circ} \mathrm{C}$ denaturation step for $10 \mathrm{~min}$, followed by 40 cycles of $95^{\circ} \mathrm{C}$ for $15 \mathrm{~s}$ and $60^{\circ} \mathrm{C}$ for $1 \mathrm{~min}$.

\section{ACKNOWLEDGMENTS}

B. P. H. J. Thomma and H. P. van Esse are supported by a Vidi and a Veni grant, respectively, of the Research Council for Earth and Life sciences of the Netherlands Organization for Scientific Research. This research was supported, in part, by the Centre for BioSystems Genomics, which is part of the Netherlands Genomics Initiative/Netherlands Organization for Scientific Research. We thank A. Kombrink for technical assistance, H. Franssen for access to the fluorescence microscope, B. Essenstam and H. Smid for excellent plant care, and K. Dobinson and B. Lievens for the kind gift of Verticillium isolates.

\section{LITERATURE CITED}

Bailey, B. A. 1995. Purification of a protein from culture filtrates of Fusarium oxysporum that induces ethylene and necrosis in leaves of Erythroxylum coca. Phytopathology 85:1250-1255.

Birck, C., Damian, L., Marty-Detraves, C., Lougarre, A., Schulze-Briese, C., Koehl, P., Fournier, D., Paquereau, L., and Samama, J.P. 2004. A new lectin family with structure similarity to actinoporins revealed by the crystal structure of Xerocomus chrysenteron lectin XCL. J. Mol. Biol. 344:1409-1420.

Bhat, R. G., and Subbarao, K. V. 1999. Host range specificity in Verticillium dahliae. Phytopathology 89:1218-1225.

Bolton, M. D., and Thomma, B. P. H. J. 2008. The complexity of nitrogen metabolism and nitrogen-regulated gene expression in plant pathogenic fungi. Physiol. Mol. Pathol. 72:104-110.

Cabral, A., Oome, S., Sander, N., Kuefner, I., Nürnberger, T., and Van den Ackerveken, G. 2012. Nontoxic Nep1-like proteins of the downy mildew pathogen Hyaloperonospora arabidopsidis: repression of necrosisinducing activity by a surface-exposed region. Mol. Plant-Microbe Interact. 25:697-708.

Dallal, B. Z., Hegedus, D. D., Buchwaldt, L., Rimmer, S. R., and Borhan, M H. 2010. Expression and regulation of Sclerotinia sclerotiorum necrosis and ethylene-inducing peptides (NEPs). Mol. Plant Pathol. 11:43-53.

de Jonge, R., and Thomma, B. P. H. J. 2009. Fungal LysM effectorsextinguishers of host immunity? Trends Microbiol. 17:151-157.

de Jonge, R., van Esse, H. P., Kombrink, A., Shinya, T., Desaki, Y., Bours, R., van der Krol, S., Shibuya, N., Joosten, M. H. A. J., and Thomma, B. P. H. J. 2010. Conserved fungal LysM effector Ecp6 prevents chitintriggered immunity in plants. Science 329:953-955.

de Jonge, R., Bolton, M. D., and Thomma, B. P. H. J. 2011. How filamentous pathogens co-opt plants: the ins and outs of fungal effectors. Curr. Opin. Plant. Biol. 14:400-406.

de Jonge, R., van Esse, H. P., Maruthachalam, K., Bolton, M. D., Santhanam, P., Saber, M. K., Zhang, Z., Usami, T., Lievens, B., Subbarao, K. V., and Thomma, B. P. H. J. 2012. Tomato immune receptor Ve1 recognizes effector of multiple fungal pathogens uncovered by genome and RNA sequencing. Proc. Natl. Acad. Sci. U.S.A. 109:5110-5115.

Dodds, P. N., and Rathjen, J. P. 2010. Plant immunity: towards an integrated view of plant-pathogen interactions. Nat. Rev. Genet. 11:539-548.

Dong, S., Kong, G., Qutob, D., Yu, X., Tang, J., Kang, J., Dai, T., Wang, H., Gijzen, M., and Wang, Y. 2012. The NLP toxin family in Phytophthora sojae includes rapidly evolving groups that lack necrosis-inducing activity. Mol. Plant-Microbe Interact. 25:896-909.

Fellbrich, G., Romanski, A., Varet, A., Blume, B., Brunner, F., Engelhardt, S., Felix, G., Kemmerling, B., Krzymowska, M., and Nürnberger, T. 2002. NPP1, a Phytophthora-associated trigger of plant defense in parsley and Arabidopsis. Plant J. 32:375-390.

Fradin, E. F., and Thomma, B. P. H. J. 2006. Physiology and molecular aspects of Verticillium wilt diseases caused by $V$. dahliae and $V$. alboatrum. Mol. Plant Pathol. 7:71-86. 
Fradin, E. F., Zhang, Z., Juarez Ayala, J. C., Castroverde, C. C. M., Nazar, R. N., Robb, J., Liu, C.-M., and Thomma, B. P. H. J. 2009. Genetic dissection of Verticillium wilt resistance mediated 1 by tomato Ve1. Plant Physiol. 150:320-332.

Frandsen, R. J. N., Andersson, J. A., Kristensen, M. B., and Giese, H. 2008. Efficient four fragment cloning for the construction of vectors for targeted gene replacement in filamentous fungi. BMC Mol. Biol. 9:70.

Garcia, O., Macedo, J. A., Tiburcio, R., Zaparoli, G., Rincones, J., Bittencourt, L. M., Ceita, G. O., Micheli, F., Gesteira, A., Mariano, A. C., Schiavinato, M. A., Medrano, F. J., Meinhardt, L. W., Pereira, G. A., and Cascardo, J. C. 2007. Characterization of necrosis and ethylene inducing proteins (NEP) in the basidiomycete Moniliophthora perniciosa, the causal agent of witches' broom in Theobroma cacao. Mycol. Res. 111:443-455.

Gehring, C. A., and Irving, H. R. 2003. Natriuretic peptides-a class of heterologous molecules in plants. Int. J. Biochem. Cell Biol. 35:13181322.

Gijzen, M., Nürnberger, T. 2006. Nep1-like proteins from plant pathogens: recruitment and diversification of the NPP1 domain across taxa. Phytochemistry 67:1800-1807.

Houterman, P. M., Cornelissen, B. J., and Rep, M. 2008. Suppression of plant resistance gene-based immunity by a fungal effector. PLoS Pathog. 4:e1000061. Published online.

Kanneganti, T. D., Huitema, E., Cakir, C., and Kamoun, S. 2006. Synergistic interactions of the plant cell death pathways induced by Phytophthora infestans Nepl-like protein PiNPP1.1 and INF1 elicitin. Mol. Plant-Microbe Interact. 19:854-863.

Klosterman, S. J., Subbarao, K. V., Kang, S., Veronese, P., Gold, S. E., Thomma, B. P. H. J., Chen, Z., Henrissat, B., Lee, Y-H., Park, J., GarciaPedrajas, M. D., Barbara, D. J., Anchieta, A., de Jonge, R., Santhanam, P., Maruthachalam, K., Atallah, Z., Amyotte, S. G., Paz, Z., Inderbitzin, P., Hayes, R. J., Heiman, D.I., Young, S., Zeng, Q., Engels, R., Galagan, J., Cuomo, C., Dobinson, K. F., and Ma, L. J. 2011. Comparative genomics yields insights into niche adaptation of plant vascular wilt pathogens. PLoS Pathog. 7:e1002137. Published online.

Kombrink, A., Sánchez-Vallet, A., and Thomma, B. P. H. J. 2011. The role of chitin detection in plant-pathogen interactions. Microbes Infect. 13:1168-1176.

Lievens, B., Brouwer, M., Vanachter, A. C. R. C., Levésque, A., Cammue, B. P. A., and Thomma B. P. H. J. 2003. Design and development of a DNA array for rapid detection and identification of multiple tomato vascular wilt pathogens. FEMS (Fed. Eur. Microbiol. Soc.) Microbiol. Lett. 223:113-122.

Ma, L. J., van der Does, H. C., Borkovich, K. A., Coleman, J. J., Daboussi, M. J., Di Pietro, A., Dufresne, M., Freitag, M., Grabherr, M., Henrissat, B., Houterman, P. M., Kang, S., Shim, W.-B., Woloshuk, C., Xie, X., Xu, J.-R., Antoniw, J. Baker, S. E., Bluhm, B. H., Breakspear, A., Brown, D. W., Butchko, R. A. E., Chapman, S., Coulson, R., Coutinho, P. M., Danchin, E. G. J., Diener, A., Gale, L. R., Gardiner, D. M., Goff, S., Hammond-Kosack, K. E., Hilburn, K., Hua-Van, A., Jonkers, W., Kazan, K., Kodira, C. D., Koehrsen, M., Kumar, L., Lee, Y.-H., Li, L., Manners, J. M., Miranda-Saavedra, D., Mukherjee, M., Park, G., Park, J., Park, S.-Y., Proctor, R. H., Regev, A., Ruiz-Roldan, M. C., Sain, D., Sakthikumar, S., Sykes, S., Schwartz, D. C., Turgeon, B. G., Wapinski, I., Yoder, O., Young, S., Zeng, Q., Zhou, S., Galagan, J., Cuomo, C. A., Kistler, H. C., and Rep, M. 2010. Comparative genomics reveals mobile pathogenicity chromosomes in Fusarium. Nature 464:367-373.

Marshall, R., Kombrink, A., Motteram, J., Loza-Reyes, E., Lucas, J., Hammond-Kosack, K. E., Thomma, B. P. H. J., and Rudd, J. J. 2011. Analysis of two in planta expressed LysM effector homologs from the fungus Mycosphaerella graminicola reveals novel functional properties and varying contributions to virulence on wheat. Plant Physiol. 156:756-769

Mattinen, L., Tshuikina, M., Mae, A., and Pirhonen, M. 2004. Identification and characterization of Nip, necrosis-inducing virulence protein of Erwinia carotovora subsp. carotovora. Mol. Plant-Microbe Interact. 17:1366-1375.

Mentlak, T. A., Kombrink, A., Shinya, T., Ryder, L. S., Otomo, I., Saitoh, H., Terauchi, R., Nishizawa, Y., Shibuya, N., Thomma, B. P. H. J., and Talbot, N. J. 2012. Effector-mediated suppression of chitin-triggered immunity by Magnaporthe oryzae is necessary for rice blast disease. Plant Cell 24:322-335.

Motteram, J., Kufner, I., Deller, S., Brunner, F., Hammond-Kosack, K. E.,
Nürnberger, T., and Rudd, J. J. 2009. Molecular characterization and functional analysis of MgNLP, the sole NPP1 domain-containing protein, from the fungal wheat leaf pathogen Mycosphaerella graminicola. Mol. Plant-Microbe Interact. 22:790-799.

Ottmann, C., Luberacki, B., Kufner, I., Koch, W., Brunner, F., Weyand, M., Mattinen, L., Pirhonen, M., Anderluh, G., Seitz, H. U., Nürnberger, T., and Oecking, C. 2009. A common toxin fold mediates microbial attack and plant defense. Proc. Natl. Acad. Sci. U.S.A. 106:1035910364.

Pemberton, C. L., and Salmond, G. P. C. 2004. The Nep1-like proteins: A growing family of microbial elicitors of plant necrosis. Mol. Plant Pathol. 5:353-359.

Pemberton, C. L., Whitehead, N. A., Sebaihia, M., Bell, K. S., Hyman, L. J., Harris, S. J., Matlin, A. J., Robson, N. D., Birch, P. R., Carr, J. P., Toth, I. K., and Salmond, G. P. 2005. Novel quorum-sensing-controlled genes in Erwinia carotovora subsp. carotovora: identification of a fungal elicitor homologue in a soft-rotting bacterium. Mol. Plant-Microbe Interact. 18:343-353.

Qutob, D., Kemmerling, B., Brunner, F., Küfner, I., Engelhardt, S., Gust, A. A., Luberacki, B., Seitz, H. U., Stahl, D., Rauhut, T., Glawischnig, E., Schween, G., Lacombe, B., Watanabe, N., Lam, E., Schlichting, R., Scheel, D., Nau, K., Dodt, G., Hubert, D., Gijzen, M., and Nürnberger, T. 2006. Phytotoxicity and innate immune responses induced by Nep1like proteins. Plant Cell 18:3721-3744.

Ruzvidzo, O., Donaldson, L., Valentine, A., and Gehring, C. A. 2011. The Arabidopsis thaliana natriuretic peptide AtPNP-A is a systemic regulator of leaf dark respiration and signals via the phloem. J. Plant Physiol. 168:1710-1714.

Santhanam, P. 2012. Random insertional mutagenesis in fungal genomes to identify virulence factors. Methods Mol. Biol. 835 509-517.

Santhanam. P., and Thomma, B. P. H. J. 2013. Verticillium dahliae Sge1 differentially regulates expression of candidate effector genes. Mol Plant-Microbe Interact. 26:249-256.

Schouten, A., van Baarlen, P., and van Kan, J. A. 2008. Phytotoxic Nep1-like proteins from the necrotrophic fungus Botrytis cinerea associate with membranes and the nucleus of plant cells. New Phytol. 177:493-505.

Shimada, T. L., Shimada, T., and Hara-Nishimura, I. 2010. A rapid and non-destructive screenable marker, FAST, for identifying transformed seeds of Arabidopsis thaliana. Plant J. 61:519-528.

Staats, M., van Baarlen, P., Schouten, A., and van Kan, J. A. L. 2007. Functional analysis of NLP genes from Botrytis elliptica. Mol. Plant Pathol. 8:209-214.

Stergiopoulos, I., Kourmpetis, Y. A., Slot, J. C., Bakker, F. T., De Wit, P. J., and Rokas, A. 2012. In silico characterization and molecular evolutionary analysis of a novel superfamily of fungal effector proteins. Mol. Biol. Evol. 29:3371-3384.

Takken, F., and Rep, M. 2010. The arms race between tomato and Fusarium oxysporum. Mol. Plant Pathol. 11:309-314.

Thomma, B. P. H. J., Nürnberger, T., and Joosten, M. H. A. J. 2011. Of PAMPs and effectors: the blurred PTI-ETI dichotomy. Plant Cell 23:415.

Wang, J. Y., Cai, Y., Gou, J. Y., Mao, Y. B., Xu, Y. H., Jiang, W. H., and Chen, X. Y. 2004. VdNEP, an elicitor from Verticillium dahliae, induces cotton plant wilting. Appl. Environ. Microbiol. 70:4989-4995.

Wu, T. D., and Nacu, S. 2010. Fast and SNP-tolerant detection of complex variants and splicing in short reads. Bioinformatics 26:873-881.

Wu, T. D., and Watanabe, C. K. 2005. GMAP: a genomic mapping and alignment program for mRNA and EST sequences. Bioinformatics 21:1859-1875.

Zhou, B. J., Jia, P. S., Gao, F., and Guo, H. S. 2012. Molecular characterization and functional analysis of a necrosis- and ethylene-inducing, protein-encoding gene family from Verticillium dahliae. Mol. PlantMicrobe Interact. 25:964-975.

\section{AUTHOR-RECOMMENDED INTERNET RESOURCES}

Broad Institute Verticillium group database:

www.broadinstitute.org/annotation/genome/verticillium_dahliae

Center for Biological Sequence Analysis SignalP3.0 database: www.cbs.dtu.dk/services/SignalP-3.0

HMMER alignment search database: hmmer.janelia.org

Sanger Wellcome Trust Pfam database: pfam.sanger.ac.uk 